\title{
Utilización de un dispositivo móvil de tecnología electrónica para tamizaje de fibrilación auricular. Estudio piloto
}

\author{
Dra. Virginia Estragó1, Br. Matías Muñoz², PhD. Ramón Álvarez-Vaz³, \\ Dra. Ximena Reyes ${ }^{4}$, Dr. Walter Reyes Caorsi ${ }^{5}$
}

\section{Resumen}

La fibrilación auricular (FA) es un problema de salud pública que genera mortalidad y morbilidad, con su mayor impacto en mayores de 65 años. Su detección es especialmente relevante para la población de riesgo. Este estudio piloto propone valorar la utilidad de un dispositivo móvil de tecnología electrónica (DMTE) para el tamizaje de FA.

Objetivo primario: evaluar la validez y confiabilidad de un DMTE para identificar FA.

Objetivo secundario: validar los procesos de recolección, transmisión, almacenamiento, procesamiento e interpretación de los datos obtenidos.

Método: los asistentes a 5 talleres del Plan Ibirapitá (entre el 15 de octubre y el 30 de noviembre de 2018) fueron invitados a participar del estudio. Se utilizó un DMTE (sensor KardiaMobile de AliveCor ${ }^{\circledR)}$ cuyo registro se contrastó con el trazado del ECG de 12 derivaciones realizado en forma casi simultánea. Se compararon los diagnósticos automáticos con los realizados por dos cardiólogos expertos de forma independiente, a partir de la lectura del registro realizado con el DMTE y de un ECG de 12 derivaciones.

Resultados: participaron del estudio 114 beneficiarios del Plan Ibirapitá, 78 del sexo femenino. La edad media fue de 72,5 años (SD 5,36; rango 53-87 años). La sensibilidad para el diagnóstico de FA del DMTE fue de 100\%, con una especificidad de $96,6 \%$. (VPP $=57,1 \%$ VPN = 100\%) y una proporción de diagnóstico correcto de 96,8\%. El DMTE catalogó como "sin clasificar" al 18,4\% de los trazados. Considerando esto, la proporción de diagnóstico correcto disminuyó a 78,9\%, sin presentar falsos negativos.

Conclusiones: el cribado de FA con la utilización de un DMTE en una población de adultos mayores es factible y confiable. El hallazgo de un 18,4\% de trazados con diagnóstico automático "sin clasificar" hace necesario contar con recursos humanos calificados para realizar la confirmación diagnóstica en esos registros.

Palabras clave: $\quad$ FIBRILACIÓN AURICULAR

TAMIZAJE

TELEMEDICINA

PREVENCIÓN CARDIOVASCULAR

\section{Use of a mobile electronic technology device for atrial fibrillation screening. Pilot study}

\section{Summary}

Atrial fibrillation $(\mathrm{AF})$ is a public health problem generating important morbidity and mortality mainly in $\geq 65$ years old population. Detection is key in the population at risk. This pilot study was designed to assess a mobile electronic technology device (METD) usefulness in AF screening.

Objective: evaluate validity and reliability of a METD in AF identification.

Secondary objective: to validate the process of collection, transmission, storage, method and interpretation of obtained data.

1. Cardióloga Comisión Honoraria para la Salud Cardiovascular.

2. Auxiliar en estadística Comisión Honoraria para la Salud Cardiovascular.

3. Profesor Agregado. Director del IESTA, FCEA, Udelar.Matías Muñoz:

4. Médico Comisión Honoraria para la Salud Cardiovascular.

5. Cardiólogo. Secretario Comisión Directiva Comisión Honoraria para la Salud Cardiovascular.

Correspondencia: Dra. Virginia Estragó. Correo electrónico: vikyestrago@gmail.com

Los autores declaran no tener conflictos de interés.

Recibido Abr 7, 2021; aceptado Jun 18, 2021 
Method: participants in 5 Ibirapitá Plan workshops (October 15-November 30, 2018) were invited to participate in the study. A KardiaMobile Alive Cor ${ }^{\circledR}$ METD was used, whose ECG recording was compared with a 12L ECG taken almost at the same time. Automatic METD report was compared with 2 independent cardiologists report considering the METD recording and the 12L ECG.

Results: 114 participants (78 female), mean age 72,5 y.o. (SD 5,36; range 53-87) were included. METD sensitivity for AF diagnosis was $100 \%$ with a $96.6 \%$ specificity $(\mathrm{PPV}=57,1 \% \mathrm{NPV}=100 \%)$, and a $96.8 \%$ number of correct diagnosis. A $18.4 \%$ of recordings were catalogued as "unclassified" by the METD. Considering this, the proportion of correct diagnosis decreased to $78.9 \%$; there were not false negatives.

Conclusions: AF screening with a METD in an older population is feasible and reliable. The finding of 18.4\% METD recordings as "unclassified" raises the needs for experts review during diagnosis confirmation.

Key words: $\quad$ ATRIAL FIBRILLATION

SCREENING

TELEMEDICINE

CARDIOVASCULAR PREVENTION

\section{Uso de dispositivo de tecnologia eletrônica móvel para rastreamento de fibrilação atrial. Estudo piloto}

A fibrilação atrial (FA) é um problema de saúde pública que gera mortalidade e morbidade, com maior impacto em pessoas com mais de 65 anos. Sua detecção é especialmente relevante para a população de risco. Este estudo piloto teve como objetivo avaliar a utilidade de um dispositivo móvel de tecnologia eletrônica (DMTE) para o monitoramento da FA.

Objetivo principal: avaliar a validade e confiabilidade de um DMTE para identificar FA.

Objetivo secundário: validar os processos de coleta, transmissão, armazenamento, processamento e interpretação dos dados obtidos.

Método: participantes de 5 oficinas do Plano Ibirapitá (entre 15 de outubro e 30 de novembro de 2018) foram convidados a participar do estudo. Foi utilizado um DMTE (sensor AliveCor® KardiaMobile), cujo registro foi contrastado com o traçado do ECG de 12 derivações realizado quase simultaneamente. Os diagnósticos automatizados foram comparados com aqueles realizados por dois cardiologistas especialistas independentes, com base na leitura gráfica do DMTE e no ECG de 12 derivações.

Resultados: participaram do estudo 114 beneficiários do Plano Ibirapitá, sendo 78 mulheres. A média de idade foi de 72,5 anos (DP 5,36; variação de 53-87 anos). A sensibilidade para o diagnóstico de FA no DMTE foi de 100\% com especificidade de $96,6 \%$. (VPP $=57,1 \%$ VPN $=100 \%$ ) e proporção de diagnóstico correto de 96,8\%. O DMTE definiu 18,4\% dos registros como "não classificados". Portanto, a proporção de diagnósticos corretos diminuiu para 78,9\% e não houve falsos negativos.

Conclusões: a triagem para FA por meio de DMTE em uma população idosa é viável e confiável. A constatação de 18,4\% dos registros com diagnóstico automático "não classificado" torna necessária a existência de recursos humanos qualificados para a realização da confirmação diagnóstica.

Palavras chave: FIBRILAÇÃO ATRIAL

TRIAGEM

TELEMEDICINA

PREVENÇÃO CARDIOVASCULAR

\section{Introducción}

La fibrilación auricular (FA) es la arritmia más frecuente en el adulto. Según el informe del "Global Burden of Diseases, Injuries, and Risk Factors", 33,5 millones de individuos en el mundo presentan FA, aproximadamente el $0,5 \%$ de la población ${ }^{(1)}$. Además, su incidencia y prevalencia están en aumento. En un seguimiento de 50 años de su cohorte, el estudio Framingham encontró que entre los períodos 1958-1967 y 1998-2007 se cuadriplicó la prevalencia de FA ajustada por edad ${ }^{(2)}$.

La FA aumenta el riesgo de muerte, provoca insuficiencia cardíaca, infarto de miocardio y accidente cerebro vascular (ACV), además de comprometer la calidad de vida de los pacientes. Su prevalencia y riesgos aumentan con la edad ${ }^{(3,4)}$. La consecuencia más temida es el ACV. Estudios contemporáneos muestran que entre un $20 \%$ y $30 \%$ de los pacientes con ictus isquémico tienen un diagnóstico de FA antes, durante o después del evento inicial ${ }^{(5)}$. A nivel nacional, un estudio publicado en $2014^{(6)}$ reportó la prevalencia de FA analizando el ECG de adultos mayores de 18 años usuarios de una institución de asistencia médica colectiva de Montevideo. Este estudio encontró una prevalencia global de FA de $2 \%$, es infrecuente antes de los 60 años y alcanza un porcentaje de 5,0\% entre los 60 y los 79 años y de $12,3 \%$ en los mayores de esa edad.

La FA puede cursar en forma asintomática y su primer registro diagnóstico puede realizarse en el contexto de un $\mathrm{ACV}^{(7,8)}$. También puede ser intermitente. El riesgo de desarrollo de ACV no está vin- 


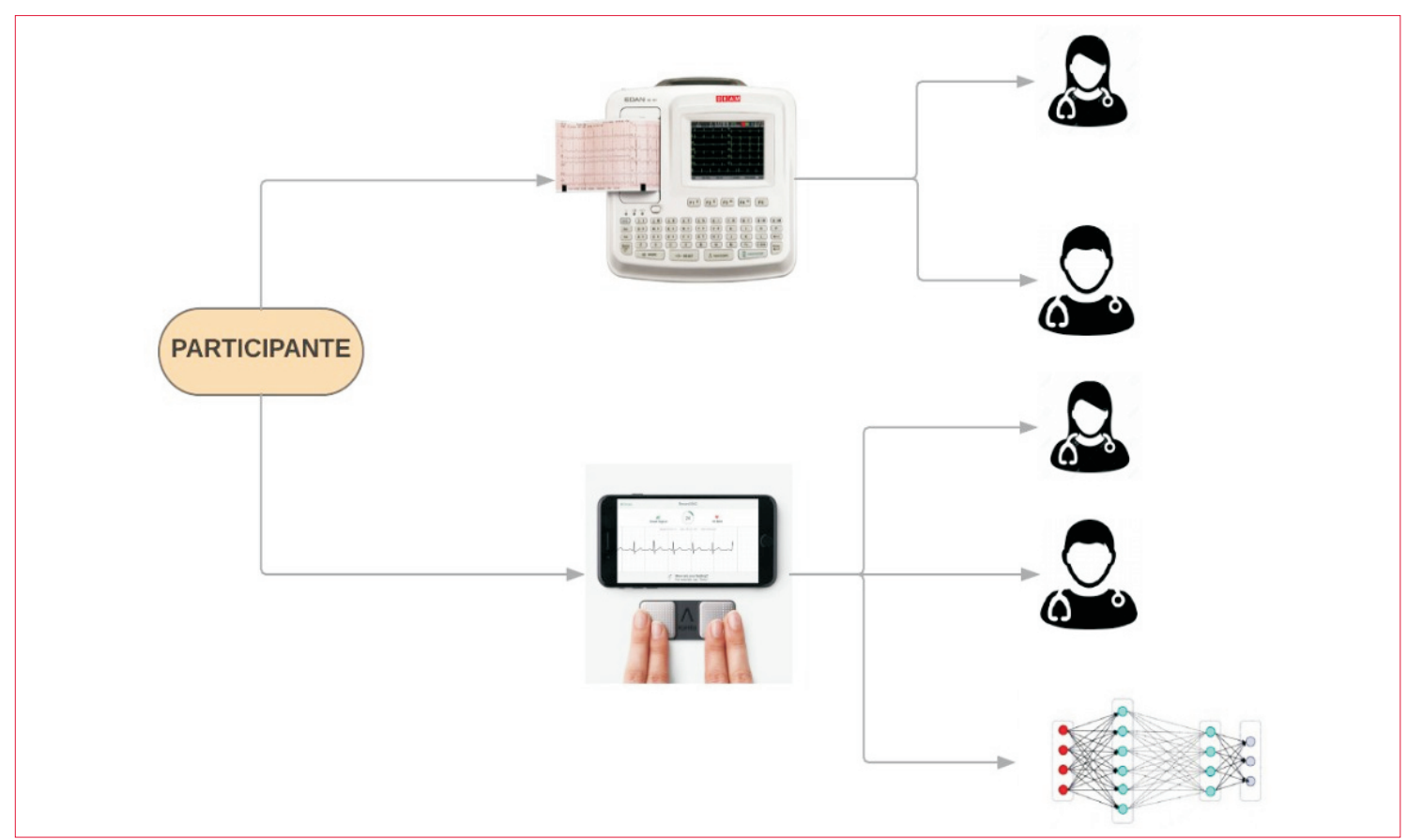

Figura 1. A cada participante se le hacen dos registros: uno con un ECG de 12 derivaciones (a) y otro con el DMTE (b). La definición del diagnóstico electrocardiográfico es llevada a cabo por dos cardiólogos en forma independiente. De esta forma, cada trazado del ECG de 12 derivaciones tiene 2 asignaciones diagnósticas (c y d), en tanto cada registro del DMTE tiene 3 asignaciones diagnósticas que corresponden, una, al diagnóstico automático del DMTE (g) y las otras 2, al diagnóstico realizado por cada uno de los cardiólogos (e y f).

culado a la presencia o ausencia de síntomas ni a su curso temporal ${ }^{(9-11)}$.

Hacer el cribado de FA es relevante desde el punto de vista clínico, epidemiológico y para la salud públi$\mathrm{ca}^{(12)}$. Se pueden emplear distintas estrategias de tamizaje: oportunístico, con un registro único (single point screening), o sistemático, con registros repetidos. Se han utilizado múltiples métodos como la toma de pulso, un ECG, el registro de dispositivos implantables (cardiodesfibriladores, marcapasos, registradores de eventos implantables), aplicaciones para teléfonos celulares vinculadas o no a relojes inteligentes usando pletismografía, parches o dispositivos para monitorización a distancia, entre una amplia variedad de opciones tecnológicas disponibles ${ }^{(13)}$.

Existe acuerdo en que la FA detectada a través de tamizaje (con cualquiera de los métodos) no es una condición benigna. En estos pacientes es muy común la concomitancia de otros factores de riesgo para ACV, lo cual justifica la planificación de estrategias de cribado ${ }^{(14)}$.

Contar con un método de detección que sea rápido, accesible, inocuo, de bajo costo y que pueda superar barreras geográficas es especialmente relevante para la población de riesgo. El propósito de este estudio piloto es valorar la utilidad de un dispositivo móvil de tecnología electrónica (DMTE), disponible comercialmente para uso individual, para el tamizaje de FA en una población de riesgo.
El objetivo primario es evaluar la validez y confiabilidad de un DMTE para identificar FA en un grupo de voluntarios adultos mayores beneficiarios del Plan Ibirapitá.

Los objetivos secundarios son validar los procesos de recolección, transmisión, almacenamiento, procesamiento e interpretación de los datos obtenidos.

\section{Método}

Se utilizó un DMTE cuyo registro se contrastó con el trazado del ECG de 12 derivaciones realizado en forma casi simultánea. El DMTE consiste en un sensor KardiaMobile de AliveCor ${ }^{\circledR}$ vinculado con una aplicación Kardia (Alivecor, Inc.) que se instala en smartphones, aprobado por la Food and Drug Administration de Estados Unidos para uso individual con este propósito. El sistema detecta una única derivada del ECG haciendo registros de 30 segundos de duración. El algoritmo diagnóstico incorporado al sistema se basa en definir la regularidad o irregularidad del ritmo y genera tres posibles diagnósticos automáticos: normal, posible FA o sin clasificar. Se compararon los diagnósticos automáticos con los llevados a cabo por dos cardiólogos expertos de forma independiente, a partir de la lectura del registro realizado con el DMTE y de un ECG de 12 derivaciones (figura 1). En los trazados dudosos, se solicitó la opinión de un tercer revisor especialista en arritmias. 


\begin{tabular}{lccc}
\multicolumn{4}{l}{ Tabla 1. Características de los participantes. } \\
\hline & Femenino & Masculino & Total \\
\hline HTA & $43(55 \%)$ & $26(72 \%)$ & $69(61 \%)$ \\
Diabetes & $7(9 \%)$ & $8(22 \%)$ & 15 \\
ACV & 0 & $4(11 \%)$ & 4 \\
C. isquémica & $3(4 \%)$ & $6(17 \%)$ & 9 \\
\hline
\end{tabular}

Tabla 2. Matriz de confusión: Diagnóstico automático del DMTE según diagnóstico de cardiólogos (excluye los trazados con diagnóstico "sin clasificar").

\begin{tabular}{lccc}
\hline & Posible FA & Normal & Total \\
\hline Con FA & 4 & 0 & 4 \\
Sin FA & 3 & 86 & 89 \\
Total & 7 & 86 & 93 \\
\hline
\end{tabular}

\section{Plan Ibirapitá}

Para priorizar la detección en los adultos mayores, la población de mayor riesgo y de menores recursos, se vinculó al Plan Ibirapitá en este trabajo de investigación. Ibirapitá se creó en el año 2015 para promover la inclusión digital de las personas mayores. Realiza talleres de capacitación y pone a disposición de sus usuarios el soporte tecnológico necesario (tabletas). En el contexto de los talleres que organiza el Plan, los participantes fueron invitados a intervenir en el estudio.

Entre el 15 de octubre y el 30 de noviembre de 2018 se llevaron a cabo 5 talleres, con 20 a 25 participantes cada uno, en el local de la Comisión Honoraria para la Salud Cardiovascular. Se elaboró un cuestionario sobre factores de riesgo para desarrollo de FA y conocimiento de presencia de esta arritmia, que quienes accedían debían contestar. Se aseguró la confidencialidad de los datos y los participantes firmaron consentimiento informado.

Se hizo la devolución del diagnóstico a cada uno de los participantes vía correo electrónico, adjuntando una copia del trazado del dispositivo, así como la recomendación de consulta a su médico de cabecera con esta información, en el caso que se considerara necesario.

\section{Análisis estadístico}

Se hicieron tablas, medidas de resumen para las variables en estudio y se calcularon valores predictivos positivos y negativos, sensibilidad y especificidad. El análisis de los datos se realizó utilizando el lenguaje de programación y software estadístico $R$ (versión 3.6.2) [R Core Team (2019). R: A language and environment for statistical computing. R Foundation for Statistical Computing, Vienna, Austria. URL https://www.R-project.org/.]
Tabla 3. Diagnóstico automático del DMTE según diagnóstico de cardiólogos (incluye los trazados con diagnóstico "sin clasificar").

\begin{tabular}{lcccc}
\hline & Posible FA & Normal & Sin clasificar & Total \\
\hline Con FA & 4 & 0 & 0 & 4 \\
Sin FA & 3 & 86 & 21 & 110 \\
Total & 7 & 86 & 21 & 114 \\
\hline
\end{tabular}

\section{Resultados}

Participaron del estudio 114 beneficiarios del Plan Ibirapitá, 78 del sexo femenino. La edad media fue de 72,5 años (SD 5,36; rango 53-87 años).

Las características de los participantes de acuerdo con los datos proporcionados por ellos se describen en la tabla 1 .

Dos cardiólogos realizaron diagnóstico de FA en 4 participantes con el análisis del ECG y del registro del DMTE en forma independiente con una concordancia en el diagnóstico de 100\%. De estos 4 participantes, solo 1 conocía que la tenía.

En 7 casos el DMTE diagnosticó posible FA. Este diagnóstico fue confirmado en $4 / 7$ casos con el análisis de los cardiólogos. En los otros 3 casos diagnosticados por el DMTE como posible FA esta no fue confirmada (falsos positivos). No se registraron falsos negativos. En consecuencia, la sensibilidad para el diagnóstico de FA del DMTE es de $100 \%$ con una especificidad de $96,6 \%(\mathrm{VPP}=57,1 \%, \mathrm{VPN}=100 \%)$ y una proporción de diagnóstico correcto de $96,8 \%$ (tabla 2 ).

El DMTE catalogó como "sin clasificar" 21 $(18,4 \%)$ trazados (tabla 3$)$. Considerando esto, la proporción de diagnóstico correcto disminuye a $78,9 \%$. Ninguno de los trazados que el dispositivo clasifica como "sin clasificar" fue FA. Esto fue corroborado por dos cardiólogos independientes y también por un tercer revisor especialista en arritmias cardíacas. Por lo tanto, en este estudio piloto el DMTE utilizado no presentó falsos negativos tanto cuando es capaz de hacer un diagnóstico automático 
como cuando no lo hace (trazados con diagnóstico automático "sin clasificar").

Utilizando un DMTE se logró una correcta adquisición de trazados de corta duración de una sola derivada que se descargan como archivo PDF. Estos archivos PDF se compartieron con los cardiólogos que participaron de este estudio para su interpretación a distancia. Se enviaron estos registros vía mail a los participantes, quienes podían compartirlos con su médico tratante para la toma de decisiones si así lo querían. De esta forma se logran completar los procesos de recolección, almacenamiento, procesamiento e interpretación de los datos obtenidos.

\section{Discusión}

En este estudio piloto, utilizando un DMTE para la detección de FA en una población de adultos mayores de bajos recursos, se encontró una sensibilidad y una especificidad comparables a las reportadas en la literatura internacional. Se lograron, asimismo, validar en esta población los procesos de recolección, transmisión, almacenamiento, procesamiento e interpretación de los datos de un DMTE diseñado para uso individual.

La FA es una arritmia de gran prevalencia, responsable de una importante morbimortalidad y en un alto porcentaje de casos es asintomática y/o intermitente. Una estrategia de detección es necesaria, fundamentalmente en la población de mayor riesgo ${ }^{(15)}$. La utilización de DMTE para la detección de FA fue estudiada y validada en múltiples escena$\operatorname{rios}^{(16)}$ y con distintas tecnologías ${ }^{(17)}$.

Una revisión sistemática de la literatura evaluó el valor del tamizaje oportunístico de FA con un registro único en el tiempo (mediante ECG o toma de pulso) para la identificación de pacientes que se podrían beneficiar con el uso de anticoagulantes para la prevención de $\mathrm{ACV}^{(18)}$. Incluyó 30 estudios (n: 122,571; edad media: 64 años; 9 países) y la prevalencia global de FA encontrada fue de $2,3 \%$, siendo de 4,4\% en los mayores de 65 años. La incidencia de FA desconocida fue $1,4 \%$ en mayores de 65 años y el $67 \%$ de estos pacientes tenían alto riesgo de ACV.

Las guías de práctica clínica recomiendan hacer tamizaje de $\mathrm{FA}^{(15)}$, pero el significado pronóstico de los individuos en los que se lleva a cabo la detección de FA aún es incierto. Un estudio reciente reporta los resultados del cribado oportunístico, realizado en 7.107 personas mayores de 65 años en las farmacias, utilizando un dispositivo manual que registra una derivada del ECG. En el seguimiento a un año de los individuos en los que se detectó FA no conoci- da $(3,6 \%)$ se encontró un exceso de mortalidad comparado con los individuos sin $\mathrm{FA}^{(19)}$.

El tamizaje sistemático es tan efectivo como el oportunístico para la detección de FA no conocida (1,64\% oportunístico vs. 1,62\% sistemático). Sin embargo, el tamizaje oportunístico tiene una mejor ecuación costo-efectividad, dado el costo de realizar ECG de 12 derivaciones y las dificultades de enrolamiento, aproximadamente $50 \%$ de los pacientes efectivamente concurren a hacerse un ECG ${ }^{(20)}$. Estos aspectos deben ser tenidos en cuenta cuando se planifica una estrategia de detección poblacional de FA.

Utilizando la misma tecnología empleada en este estudio, Orchard y colaboradores ${ }^{(12)}$ logran demostrar la factibilidad de la detección de FA a nivel comunitario, comprobando que es de fácil aplicación y aceptado por la población. Durante el período de vacunación antigripal en Sidney, Australia, en 2015, en 5 consultorios de medicina general se registró con el monitor AliveCor el ECG a 2.476 pacientes mayores de 65 años. El registro luego era revisado por dos cardiólogos. La sensibilidad del dispositivo para la detección de FA fue de $95 \%$ y la especificidad de $99 \%$. Los trazados catalogados como «sin clasificar» $(8,4 \%)$ se relacionaban en su mayoría a bradicardia o taquicardia sinusal ${ }^{(21)}$. En nuestro estudio reportamos un $18,4 \%$ de registros con diagnóstico automático "sin clasificar". Otro reporte en el que se utilizó el AliveCor, el estudio iREAD reclutó en forma prospectiva 52 pacientes con FA que se hospitalizaban para el inicio de drogas antiarrítmicas, reportando una sensibilidad del $100 \%$ y especificidad del 89,2\% para el diagnóstico de FA y un $27,6 \%$ de registros sin clasificar ${ }^{(22)}$. También con el mismo DMTE, otra investigación realizada en Kenia que incluyó 50 pacientes que consultaron en forma consecutiva en un lapso de 2 semanas en el Hospital Kijabe, mostró que la detección de FA en el contexto de un hospital de bajos recursos es factible, tiene un buen rendimiento diagnóstico una vez que los trazados son revisados por cardiólogos y es bien aceptado por los participantes ${ }^{(16)}$.

El DMTE que utilizamos en este estudio fue también empleado para validar la exactitud en el diagnóstico de un reloj inteligente, que usa pletismografía y se vincula a la aplicación KardiaMobile en el celular, en una cohorte prospectiva de 672 pacientes hospitalizados. Este estudio encuentra que el diagnóstico de FA por fotopletismografía es factible y seguro, pero una de sus limitaciones es una alta tasa de registros de mala calidad que no permiten definir un diagnóstico(23).

El estudio hecho por la Universidad de Stanford y financiado por la empresa Apple Inc. es un estudio icónico en el uso de la tecnología para la detección de 
FA a distancia ${ }^{(24)}$. Al igual que en el estudio previo, se utiliza un reloj inteligente que detecta, a través de fotopletismografía, la presencia de pulso regular o irregular. El reloj (iWatch) está vinculado a un celular inteligente (iPhone) con la aplicación KardiaMobile. Cuando detecta pulso irregular, envía una notificación al participante y se le ofrece una teleconsulta a través de un servicio de telesalud. Una vez que se constata que el participante cumple con los criterios de elegibilidad (mayor de 22 años, no tener FA conocida, no estar usando anticoagulantes), se le envía por correo un parche para registro del ECG que debe usar por al menos 7 días. De los 419.297 participantes el 0,52\% (2.161 participantes) recibió notificación de pulso irregular. De estos, 450 devolvieron los parches con registros pasibles de ser analizados. Los autores concluyen que la probabilidad de recibir notificación de pulso irregular fue baja. En los pacientes que recibieron notificación de pulso irregular, 34\% tenía FA. Estos hallazgos no sorprenden, dado que los participantes eran personas mayoritariamente jóvenes con una media de $41 \pm 13$ años, siendo la proporción de participantes mayores de 65 años de 5,9\%. La prevalencia de FA detectada en los distintos estudios de tamizaje de FA depende de la población incluida, así como del tiempo de registro (por ejemplo, un solo registro, varios registros puntuales, dispositivos de monitorización continua, etc. $)^{(18,25-27)}$.

Un aspecto relevante del estudio de Perez y Mahaffey y colaboradores ${ }^{(24)}$ radica en que logra mostrar las enormes posibilidades que puede ofrecer la tecnología para la atención en salud, destacando que todas las fases del estudio se realizaron a distancia, desde la selección de los participantes, la firma de consentimiento informado, la consulta médica por telemedicina con la derivación a un centro asistencial en el caso de hallazgo de alguna arritmia grave, el envío y la devolución de los parches para el registro electrocardiográfico por correo, etcétera.

Las tecnologías de la información y la comunicación (TICs) han generado una revolución a nivel mundial, también en la medicina y ciencias afines. Su desarrollo ha contribuido a lo que hoy se denomina telemedicina o telesalud ${ }^{(28,29)}$. En este contexto de pandemia y quizás de ahora en adelante, disponer de un recurso accesible y sencillo de utilizar que permita hacer a distancia el tamizaje, un eventual diagnóstico y probablemente el inicio de un tratamiento, así como el seguimiento de una patología de trascendencia clínica como la FA, será de enorme utilidad para prevenir complicaciones, evitar traslados innecesarios y optimizar la asistencia.

\section{Conclusiones}

El tamizaje de FA con un DMTE, en una población de adultos mayores de bajos recursos es factible, tuvo buena aceptación y logró identificar FA con una sensibilidad y especificidad comparables a las reportadas en la literatura internacional.

Entre las limitaciones del estudio se encuentra el bajo número de participantes y la presencia de un claro sesgo femenino.

El hallazgo de un 18,4\% de trazados con diagnóstico automático "sin clasificar» hace necesario contar con recursos humanos calificados para realizar la confirmación diagnóstica en esos registros.

Un contexto de pandemia jerarquiza la importancia de estos métodos.

\section{Agradecimiento}

A los Dres. Mario Zelarayán y Bernardo Pérez por su invalorable apoyo en esta investigación.

\footnotetext{
Virginia Estragó, ORCID 0000-0002-8208-8784 . . Matías Muñoz, ORCID 0000-0002-2397-863X.

Ramón Álvarez, ORCID 0000-0002-2505-4238.

Ximena Reyes, ORCID 0000-0003-1988-6799.

Walter Reyes, ORCID 0000-0002-4670-5765.
}

\section{Bibliografía}

1. Chugh SS, Roth GA, Gillum RF, Mensah GA. Global burden of atrial fibrillation in developed and developing nations. Glob Heart. 2014;9(1):113-9. doi: 10.1016/j.gheart.2014.01.004.

2. Schnabel RB, Yin X, Gona $\mathbf{P}$, Larson MG, Beiser AS, McManus DD, et al. 50 year trends in atrial fibrillation prevalence, incidence, risk factors, and mortality in the Framingham Heart Study: a cohort study. Lancet Lond Engl. 2015;386(9989): 154-62. doi: 10.1016/S0140-6736(14)61774-8.

3. Lloyd-Jones DM, Wang TJ, Leip EP, Larson MG, Levy D, Vasan RS, et al. Lifetime risk for development of atrial fibrillation: the Framingham Heart Study. Circulation. 2004;110(9):1042-6. doi: 10.1161/01.CIR.0000140263.20897.42.

4. Kannel WB, Abbott RD, Savage DD, McNamara PM. Epidemiologic features of chronic atrial fibrillation: the Framingham study. N Engl J Med. 1982;306(17):1018-22. doi: 10.1056/NEJM198204293061703.

5. Kishore A, Vail A, Majid A, Dawson J, Lees KR, Tyrrell PJ, et al. Detection of atrial fibrillation after ischemic stroke or transient ischemic attack. Stroke. 2014;45(2):520-6. doi: 10.1161/STROKEAHA.113.003433. 
6. Sandoya E, Aguilar MP, Vázquez H. Prevalencia de la fibrilación auricular en la población adulta de Montevideo. Rev Urug Cardiol. 2014;29(2):187-91.

7. Boriani G, Laroche C, Diemberger I, Fantecchi E, Popescu MI, Rasmussen LH, et al. Asymptomatic atrial fibrillation: clinical correlates, management, and outcomes in the EORP-AF Pilot General Registry. Am J Med. 2015;128(5):509-18.e2. doi 10.1016/j.amjmed.2014.11.026.

8. Kirchhof $\mathbf{P}$, Benussi S, Kotecha D, Ahlsson A, Atar D, Casadei B, et al. Guía ESC 2016 sobre el diagnóstico y tratamiento de la fibrilación auricular, desarrollada en colaboración con la EACTS. Rev Esp Cardiol. 2017;20(1):50.e1-e84. 10.1016/j.recesp. 2016. 11.014

9. Xiong Q, Proietti M, Senoo K, Lip GYH. Asymptomatic versus symptomatic atrial fibrillation: A systematic review of age/gender differences and cardiovascular outcomes. Int $J$ Cardiol 2015;191:172-7. doi: 10.1016/j.ijcard.2015.05.011.

10. Lip GY, Hee FL. Paroxysmal atrial fibrillation. QJM. 2001;94:665-78. doi: 10.1093/qjmed/94.12.665.

11. Link MS, Giugliano RP, Ruff CT, Scirica BM, Huikuri H, Oto A, et al. Stroke and mortality risk in patients with various patterns of atrial fibrillation: results from the ENGAGE AF-TIMI 48 Trial (Effective Anticoagulation With Factor Xa Next Generation in Atrial Fibrillation-Thrombolysis in Myocardial Infarction 48). Circ Arrhythm Electrophysiol 2017;10:e004267. doi: 10.1161/CIRCEP.116.004267.

12. Neubeck L, Orchard J, Lowres N, Freedman SB. To screen or not to screen? examining the arguments against screening for atrial fibrillation. Heart Lung Circ. 2017;26(9):880-6. doi: 10.1016/j.hlc.2017. 05.118

13. Mairesse GH, Moran P, Van Gelder IC, Elsner C, Rosenqvist M, Mant J, et al. Screening for atrial fibrillation: a European Heart Rhythm Association (EHRA) consensus document endorsed by the Heart Rhythm Society (HRS), Asia Pacific Heart Rhythm Society (APHRS), and Sociedad Latinoamericana de Estimulación Cardíaca y Electrofisiología (SOLAECE).Europace. 2017;19(10):1589-623. doi 10.1093/europace/eux177.

14. Freedman B, Camm J, Calkins H, Healey JS, Rosenqvist M, Wang J, et al. Screening for atrial fibrillation: a report of the AF-SCREEN International Collaboration. Circulation. 2017;135(19):185167. doi: 10.1161/CIRCULATIONAHA.116.026693.

15. Hindricks G, Potpara T, Dagres N, Arbelo E, Bax JJ, Blomström-Lundqvist C, et al. 2020 ESC guidelines for the diagnosis and management of atrial fibrillation developed in collaboration with the European Association of Cardio-Thoracic Surgery (EACTS) the task force for the diagnosis and management of atrial fibrillation of the European Society of Cardiology (ESC) developed with the special contribution of the European Heart Rhythm Association (EHRA) of the ESC. Eur Heart J. 2021;42(5): 373-498. doi: 10.1093/eurheartj/ehaa612. Fe de errata en: Eur Heart J. 2021;42(5):546-547. doi: 10.1093/ eurheartj/ehaa945.

16. Evans GF, Shirk A, Muturi P, Soliman EZ. Feasibility of using mobile ECG recording technology to detect atrial fibrillation in low-resource settings. Glob Heart. 2017;12(4):285-9. doi: 10.1016/j. gheart.2016.12.003.

17. Brasier N, Raichle CJ, Dörr M, Becke A, Nohturfft V, Weber S, et al. Detection of atrial fibrillation with a smartphone camera: first prospective, international, two-centre, clinical validation study (DETECT AF PRO). Europace. 2019;21(1):41-7. doi: 10.1093/europace/euy176.

18. Lowres N, Neubeck L, Redfern J, Freedman SB. Screening to identify unknown atrial fibrillation. A systematic review. Thromb Haemost. 2013;110(2):213-22. doi: 10.1160/TH13-02-0165.

19. Zink MD, Mischke KG, Keszei AP, Rummey C, Freedman B, Neumann G, et al. Screen-detected atrial fibrillation predicts mortality in elderly subjects. Europace. 2020;23(1):29-38. doi: 10.1093/europace/euaa190

20. Ben Freedman S, Lowres N. Asymptomatic Atrial Fibrillation: The case for screening to prevent stroke. JAMA. 2015;314(18):1911-2. doi: 10.1001/jama. 2015.9846 .

21. Orchard J, Lowres N, Freedman SB, Ladak L, Lee W, Zwar N, et al. Screening for atrial fibrillation during influenza vaccinations by primary care nurses using a smartphone electrocardiograph (iECG): a feasibility study. Eur J Prev Cardiol. 2016;23:13-20. doi: 10.1177/2047487316670255.

22. William AD, Kanbour M, Callahan T, Bhargava M, Varma N, Rickard J, et al. Assessing the accuracy of an automated atrial fibrillation detection algorithm using smartphone technology: The iREAD Study. Heart Rhythm. 2018;15(10):1561-5. doi: 10.1016/j.hrthm.2018.06.037.

23. Dörr M, Nohturfft V, Brasier N, Bosshard E, Djurdjevic A, Gross S, et al. The WATCH AF Trial: SmartWATCHes for detection of atrial fibrillation. JACC Clin Electrophysiol. 2019;5(2):199-208. doi: 10.1016/j.jacep.2018.10.006.

24. Perez MV, Mahaffey KW, Hedlin H, Rumsfeld JS, Garcia A, Ferris T, et al. Large-scale assessment of a smartwatch to identify atrial fibrillation. $\mathrm{N}$ Engl J Med. 2019;381(20):1909-17. doi: 10.1056/ NEJMoa1901183.

25. Svennberg E, Engdahl J, Al-Khalili F, Friberg L, Frykman V, Rosenqvist M. Mass screening for untreated atrial fibrillation. Circulation. 2015;131 
(25):2176-84. doi: 10.1161/CIRCULATIONAHA. 114.014343 .

26. Kaasenbrood F, Hollander M, Rutten FH, Gerhards LJ, Hoes AW, Tieleman RG. Yield of screening for atrial fibrillation in primary care with a hand-held, single-lead electrocardiogram device during influenza vaccination. Europace. 2016;18(10): 1514-20. doi: 10.1093/europace/euv426.

27. Chan N-Y, Choy C-C. Screening for atrial fibrillation in 13122 Hong Kong citizens with smartphone electrocardiogram. Heart. 2017;103:24-31. doi: 10.1136/heartjnl-2016-309993.

28. Center for the Connected Health Policy. What is telehealt? [Internet]. CCHP. [consulta: 22 de Oct 2020]. Disponible en: https://www.cchpca.org/about/ about-telehealth

29. Reyes Caorsi W. Efectos colaterales positivos de la pandemia. Rev Urug Cardiol. 2020;35(2):155-62. doi: 10.29277/cardio.35.2.6

\section{Contribución de autores}

Virginia Estragó. Concepción y diseño del estudio, redacción del manuscrito.

Matías Muñoz. Concepción y diseño del estudio, redacción del manuscrito.

Ramón Álvarez. Concepción y diseño del estudio, análisis e interpretación de los datos, redacción del manuscrito,

Ximena Reyes. Adquisición de datos.

Walter Reyes. Revisión crítica del contenido intelectual del manuscrito.

Todos aprueban la versión adjunta del manuscrito para ser considerada para publicación 
Anexo

\section{Hoja de información}

El Plan Ibirapitá y la Comisión Honoraria para la Salud Cardiovascular lo invitan a participar del estudio "UTILIDAD DEL UN DISPOSITIVO MOVIL DE TECNOLOGÍA ELECTRÓNICA PARA EL DIAGNÓSTICO DE FIBRILACIÓN AURICULAR" cuyo objetivo es la detección de una arritmia cardíaca llamada fibrilación auricular.

Normalmente, su corazón trabaja como una bomba que lleva sangre a los pulmones y al resto del cuerpo. Para ayudar a que esto suceda, su corazón tiene un sistema eléctrico que garantiza que se contraiga de manera ordenada.

La fibrilación auricular es la aparición de una frecuencia cardíaca acelerada e irregular que puede aumentar el riesgo de sufrir un accidente cerebrovascular, insuficiencia cardíaca y otras complicaciones relacionadas con el corazón.

El diagnóstico de fibrilación auricular se realiza a través el registro del ritmo cardíaco. El registro del ritmo cardíaco puede realizarse a través de un electrocardiograma o a través de un dispositivo de tecnología electrónica móvil llamado Kardia Mobile. Este dispositivo consiste en dos almohadillas sobre las que, al apoyar los dedos durante 30 segundos, se registrará su ritmo cardíaco. Ese registro se transmite a una aplicación que archiva la información. De esta manera podemos saber si su ritmo cardíaco es normal o si tiene fibrilación auricular.

En el contexto de un taller del Plan Ibirapitá, si usted está de acuerdo en participar, se le realizará un breve cuestionario por parte de los docentes. Luego se le solicitará que apoye sus dedos en las almohadillas para realizar el registro de su ritmo cardíaco.

Estos datos serán confidenciales, es decir, no se darán a conocer los valores individuales, y se utilizarán con el propósito de la investigación actual o investigaciones futuras. En algunos casos puede ser necesario además del registro con el Kardia Mobile, realizar también un electrocardiograma.

Los datos de los registros se enviarán a una base de datos y serán analizadas po cardiólogos de la Comisión Honoraria para la Salud Cardiovascular. Si se encuentran valores fuera de rangos normales, se le comunicará a través de un mail que solicitaremos con ese fin. En dicho mail recibirá una serie de recomendaciones que lo orientarán en cómo actuar ante el hallazgo de fibrilación auricular.

Ante cualquier consulta no dude en comunicarse con nosotros

Ibirapitá

Comisión Honoraria para la Salud Cardiovascular: 24802715 interno 100 Su colaboración es muy valiosa, muchas gracias.

Consentimiento informado.

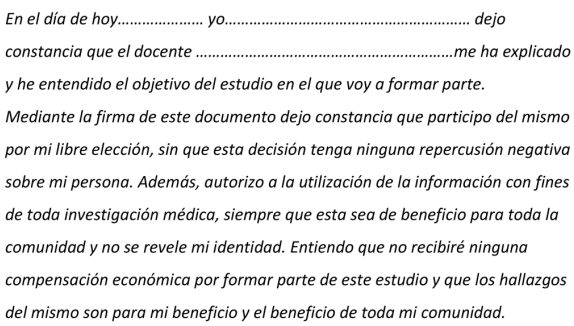

Al firmar este consentimiento acepto participar del estudio

"UTILIDAD DEL UN DISPOSITIVO DE TECNOLOGIA ELECTRONICA MOVIL PARA EL DIAGNOSTICO DE FIBRILACION AURICULAR"

FIRMA DEL VOLUNTARIO

ACLARACIÓN DE FIRMA

CI: 